\title{
SECONDARY OBSTRUCTIONS FOR FIBRE SPACES
}

\author{
BY ROBERT HERMANN ${ }^{1}$
}

Communicated by W. S. Massey, September 10, 1958

1. The aim of this note is to set up machinery for the computation of the second obstruction cohomology class to the extension of a crosssection of a fibre space (in the sense of Serre [6]), $F \rightarrow^{i} E \rightarrow^{p} B$, with base $B$ a simply connected $\mathrm{C}-\mathrm{W}$ complex, and connected fiber $F$, total space $E$, projection map $p$ and inclusion $i$. (We will use this notation below to identify the component parts of fibre spaces.) The problem is reduced to the computation of the Eilenberg-McLane invariant of $F$, just as for the obstruction problem for mapping $B \rightarrow F,[2]$, and additional twisting invariants of the fibre space. It is not yet clear how easily these latter invariants can be computed; in simple cases (for example, if $F$ is a sphere [4]), the method works very elegantly. In this example the simplifying points seem to be that (1) the second nonvanishing homotopy group of the fibre is cyclic of prime order and (2) the additive cohomology of $E$ can be explicitly determined.

2. The calculation is based on a construction introduced by J. C. Moore [5] in the category of semi-simplicial fibre spaces giving a decomposition of $E$ by successively killing off homotopy groups of the fibre.

Suppose $0<n(1)<n(2)<\cdots$ are the dimensions in which $F$ has non-zero homotopy groups. Define $A_{j}=\pi_{n(j)}(F)$. Recall that the Postnikov system of $F$ provides a tower of fibre spaces (and commutative diagrams)

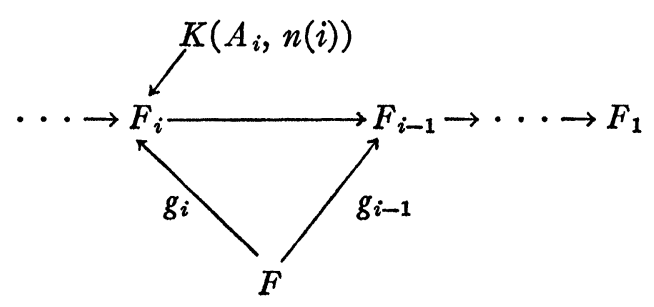

such that the map $g_{i}$ induces an isomorphism on homotopy groups in dimensions $\leqq n(i)$. (If $A$ is an abelian group, $K(A, n)$ is a space all of whose homotopy groups are zero but the one in dimension $n$, which is isomorphic to $A$.)

1 This research was supported in part by OOR, U. S. Army under contract number DA 19-020-ORD-3778. 
A decomposition of the fibre space $E \rightarrow B$ provides a tower of fibre spaces (with commutative diagrams)

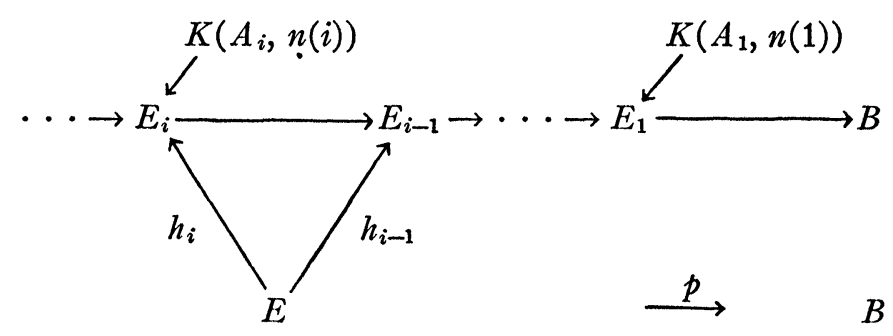

such that $h_{i}$ restricted to $F$ is $g_{i}(i=1,2, \cdots)$. Call $p_{i}$ the fibre map obtained $E_{i} \rightarrow B$. The fibre of $p_{i}$ is $F_{i}$.

As we are looking at it, geometrically, rather than from the point of view of semi-simplicial complexes, both of these constructions are not natural. The freedom in constructing the maps $h_{i}$ will be exploited to make computations. We sketch one method of constructing 2.2: Let $k^{0}(E) \in H^{n(1)+1}\left(B, A_{1}\right)$ be the characteristic class of the fibre space $F \rightarrow E \rightarrow B$, i.e. the image under transgression of the fundamental class of $H^{n(1)}\left(F, A_{1}\right)$. Let $K\left(A_{1}, n(1)\right) \rightarrow E_{1}^{p_{1}} \rightarrow B$ be a fibre space killing off $k^{0}(E) . h_{1}: E \rightarrow E_{1}$ is obtained by lifting the map $p: E \rightarrow B$ subject to the condition that $h_{1}$ restricted to $F$ is the map $g_{1} . h_{1}$ can be made into a fibre map with fibre $F_{1}^{\prime}$. One sees that $F_{1}^{\prime} \subset F$ and

$$
\pi_{j}\left(F_{1}^{\prime}\right)=\left\{\begin{array}{cl}
0 & j \leqq n(1), \\
\pi_{j}(F) & \text { if } j>n(1) .
\end{array}\right.
$$

Let $k^{1}(E) \in H^{n(2)+1}\left(E_{1}, A_{2}\right)$ be the characteristic class of the fibre space $F_{1}^{\prime} \rightarrow E \rightarrow^{h_{1}} E_{1}$, and let $E_{2}$ be a fibre space over $E_{1}$ killing off $k^{1}(E)$, etc.

3. Let $B_{j}, j=1,2, \cdots$, be the $j$-dimensional skeleton of the C-W complex $B$. Suppose $f: B_{n(j)} \rightarrow E$ is a cross-section of our fibre space $E \rightarrow B$ over the $n(j)$-skeleton. The obstruction cohomology class $w(f) \in H^{n(j)+1}\left(B, A_{j}\right)$ is defined as in [1] or [3]. If $j=1$, the first obstruction case, $w(f)$ is just the characteristic class of the fibre space $E \rightarrow B$.

We show that the higher obstruction classes can be calculated as first obstruction classes in fibre spaces obtained from the basic fibre space $E \rightarrow B$ by the above constructions.

$h_{j-1} f: B_{n(j)} \rightarrow E_{j-1}$ is a cross-section of $E_{j-1} \rightarrow B$ over the $n(j)$ skeleton. It can be extended over all of $B$ to give a cross-section $f^{\prime}: B \rightarrow E_{j-1}$. (Conversely, a cross-section $f^{\prime}: B \rightarrow E_{j-1}$ defines a cross-section of 
$E \rightarrow B$ over the $n(j)$-skeleton.) Let $k^{j-1}(E) \in H^{n(j)+1}\left(E_{j-1}, A_{j}\right)$ be the characteristic class of the fibre space $K\left(A_{j}, n(j)\right) \rightarrow E_{j} \rightarrow E_{j-1}$.

TheOREM 3.1. $w(f)=f^{\prime *}\left(k^{j-1}(E)\right)$, where $f^{\prime *}$ is the map on cohomology classes induced by $f^{\prime}$.

Proof. We have a commutative diagram

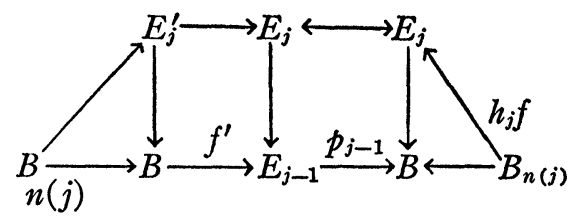

where $E_{j}^{\prime}$ is the fibre space over $B$ induced by $f^{\prime}$. It is clear from this diagram that the obstruction cohomology class to extending $h_{j} f$ is equal to $w(f)$ and is also equal the obstruction cohomology class to extending the cross-section of the fibre space $K\left(A_{j}, n(j)\right) \rightarrow E_{j}^{\prime} \rightarrow B$, (a first obstruction problem!) which is just $f^{\prime *}\left(k^{i-1}(E)\right)$.

4. Second obstruction case (i.e. $j=2$ ). Given a cross-section $f: B_{n(1)} \rightarrow E$, we make use of a more explicit construction of $E_{1}$.

There is a unique $a(f) \in H^{n(1)}\left(E, A_{1}\right)$ such that (1) $f^{*}(a(f))=0$ and (2) $a(f)$ restricted to $F$ is the fundamental class [3]. Construct a map $\alpha: E \rightarrow K\left(A_{1}, n(1)\right)$ such that $\alpha^{*}\left(i_{1}\right)=a(f) .\left(i_{1}\right.$ is the fundamental class of $K\left(A_{1}, n(1)\right)$. Define $E_{1}=B \times K\left(A_{1}, n(1)\right)$ and $h_{1}: E \rightarrow E_{1}$ as the product of $\alpha$ and $p . k^{1}(E) \in H^{n(2)+1}\left(B \times K\left(A_{1}, n(1), A_{2}\right)\right)$ is the invariant of $E$ defined in $\$ 2$.

Theorem 4.1. If $A_{2}$ is isomorphic to the integers $Z$ or the integers $\bmod l, Z_{l}$, with $l$ a prime number, $k^{1}(E)$ can be characterized by the conditions $(1) h_{1}{ }^{*}\left(k^{1}(E)\right)=0$ and $(2), k^{1}(E)$ restricted to $K\left(A_{1}, n(1)\right)$ is the Eilenberg-McLane invariant of $F$ [2] provided this invariant is not zero.

Now, let $\lambda: B \rightarrow E_{1}$ be the injection of $B$ on the first factor. Notice that $\lambda$ restricted to $B_{n(2)}$ and $h_{1} f$ are homotopic since $f^{*}\left(h_{1} *\left(i_{1}\right)\right)=0$ $=\lambda *\left(i_{1}\right)$. We have proved

THEOREM 4.2. In this second obstruction case, $w(f)=\lambda^{*}\left(k^{1}(E)\right)$.

EXAMPLE. Liao has given a formula for $w(f)$ in case $F=S_{n(1)}$, a sphere of dimension $n(1)>2$, and $E \rightarrow^{p} B$ is a fibre bundle with the orthogonal group as structural group. The formula is:

$$
p *(w(f))=S q^{2}(a(f))+p *\left(\psi\left(S q^{2}(a(f))\right) \cup a(f),\right.
$$

where $\psi: H^{n(1)+2}\left(E, Z_{2}\right) \rightarrow H^{2}\left(B, Z_{2}\right)$ is the "integration over the 
fibre" homomorphism [6, p. 470]. This formula is extended to fibre spaces in [3], but the proof is cumbersome. To prove it by our method, set $x=S q^{2}(a(f))+p *\left(\psi\left(S q^{2}(a(f))\right)\right) \cup a(f)$. One proves by the Gysin sequence that $x=p *(b)$ for some $b \in H^{n(1)+2}\left(B, Z_{2}\right)$. Then,

$$
h_{1}^{*}\left(S q^{2}\left(i_{1}\right)+\psi\left(S q^{2}(a(f))\right) \cup i_{1}+b\right)=0
$$

and, by the characterization,

$$
k^{1}(E)=S q^{2}\left(i_{1}\right)+\psi\left(S q^{2}(a(f)) \cup i_{1}+b\right.
$$

and then $\lambda^{*}\left(k^{1}(E)\right)=b=w(f)$, i.e. $p *(w(f))=x$.

In Liao's case $\psi\left(S q^{2}(a(f))\right)$ is the second Stiefel-Whitney class of the sphere bundle. In the general case one sees that it is independent of the cross-section chosen and can be considered as a twisting invariant of the fibre space, i.e. if the fibre space is isomorphic to $B \times S_{n(1)}$ the class is zero.

For a general fibre $F$, the pattern seems the same as long as $A_{2}$ is cyclic of prime order. (Unfortunately, most of the geometrically interesting examples do not satisfy this condition.) Then, $p *(w(f))$ $=\sum_{m} \theta_{m}(a(f)) \cup p *\left(b_{m}\right)$, where the $\theta_{m}$ are primary cohomology operations: $H^{*}\left(E, A_{1}\right) \rightarrow H^{*}\left(E, A_{2}\right)$, the $b_{m} \in H^{*}\left(B, A_{2}\right)$, and the cupproduct $U$ is defined by means of the ring structure on $A_{2}$. The elements $b_{m}$ whose dimensions are $>0$ represent the twisting invariants of the fibre space. If all the operations $\theta_{m}$ are additive the $b_{m}$ are independent of the section $f$ and they are zero if $E$ is a product. The explicit computation of the $b_{m}$ depends on knowing the additive cohomology of $E$. Finally, in case $E \rightarrow B$ is a fibre bundle with structural group $G$, those $b_{m}$ whose dimensions are $\leqq n(1)$ are characteristic classes of the principal bundle $G$-structure.

\section{BIBLIOGRAPHY}

1. W. Barcus, Note on cross-sections over $\mathrm{C}-\mathrm{W}$ complexes, Quart. J. of Math. Oxford vol. 5 (1954) pp. 150-160.

2. S. Eilenberg and S. McLane, On the groups $H(\pi, n)$, III, Ann. of Math. vol. 60 (1954) pp. 513-556.

3. R. Hermann, Obstruction theory in fibre spaces, to appear in Illinois J. of Math.

4. S. D. Liao, On the theory of obstructions of fibre bundles, Ann. of Math. vol. 60 (1954) pp. 146-191. 1956.

5. J. C. Moore, Seminar notes on semi-simplicial complexes, Princeton University,

6. J. P. Serre, Homologie singulière des espaces fibres, Ann. of Math. vol. 54 (1951) pp. 425-505.

\section{HARVARD University}

